Supporting Information of: Ultrasensitive all-optical thermometry using nanodiamonds with high concentration of silicon-vacancy centers and multiparametric data analysis

Sumin Choi, ${ }^{* \dagger}$ Viatcheslav N. Agafonov, ${ }^{\ddagger}$ Valery A. Davydov, $"$ and Taras Plakhotnik ${ }^{\dagger}$

$\dagger$ School of Mathematics and Physics, The University of Queensland, QLD 4072, Australia $\ddagger$ GREMAN, UMR CNRS-7347, University F. Rabelais, 37200 Tours, France

\L.F. Vereshchagin Institute for High Pressure Physics, The Russian Academy of Sciences, Troitsk, Moscow 108840, Russia

E-mail: sumin.choi@uq.edu.au 


\section{Scanning electron microscopy}

Scanning electron microscope (SEM) image of nanodiamonds is in Fig. S1. Probe-sonication was used to break down aggregates and the majority of individual nanodiamonds is less than $100 \mathrm{~nm}$ across. A round sphere is silicon residue formed during fabrication.

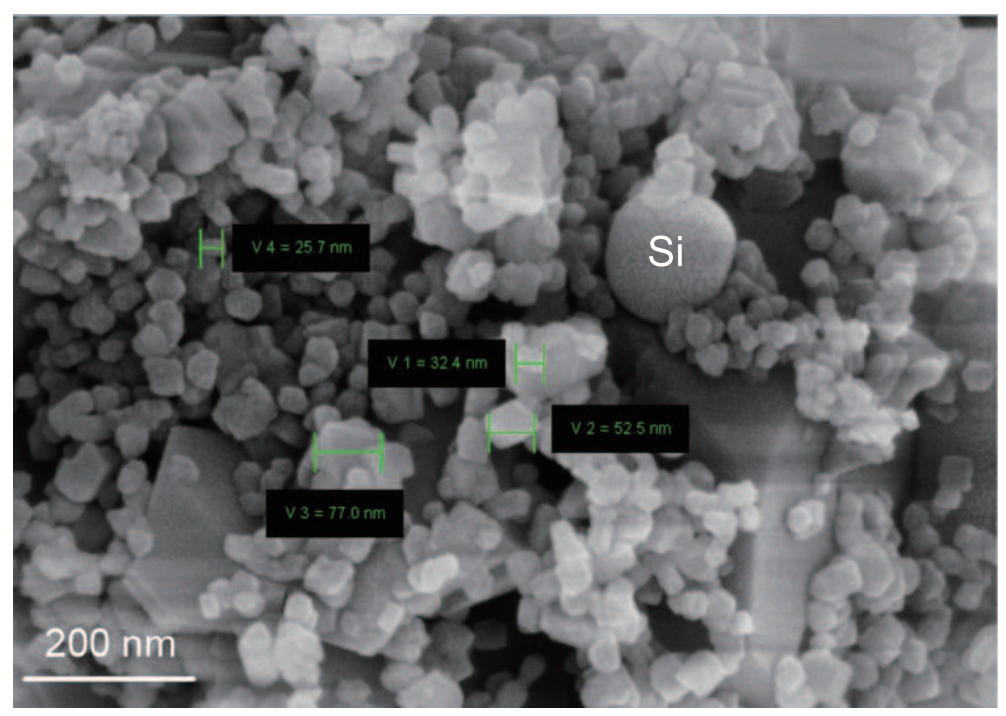

Figure S1: Scanning electron microscope (SEM) of nanodiamonds. A large size of round shape is a silicon sphere which is used as heating source. 


\section{Experimental setup}

A schematic setup of the experiment is presented in Fig. S2a. Details are described in Experimental section. Fig. S2b shows difference of heating from silicon spheres between confocal and wide-field microscopy.

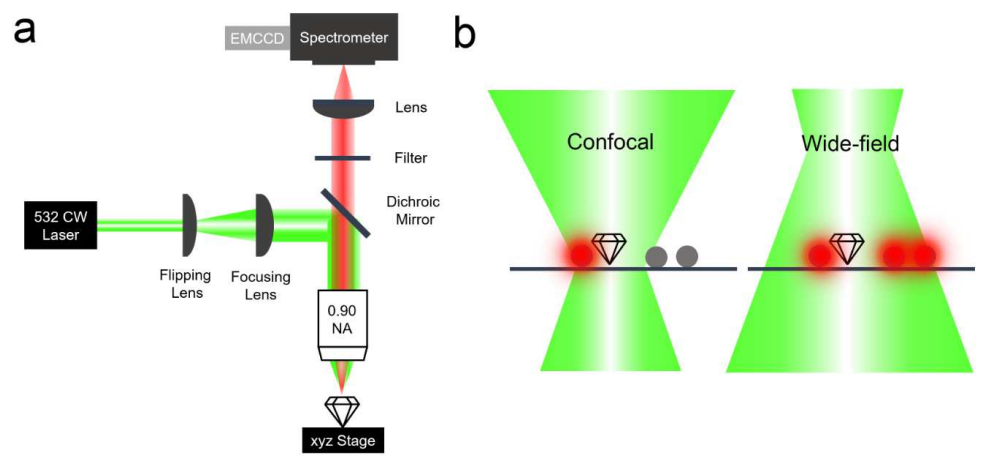

Figure S2: a. Schematic setup for imaging and photoluminescence (PL) measurements. 532-nm excitation from a continuous wave (cw) laser (Coherent. Verdi-V5) is focused onto the sample through an air-immersed objective. The emission is filtered by a dichroic mirror (Semrock Di02-R594 25x36) and a long pass filter, and then collected into the spectrometer and EMCCD. b. Diagram of laser heating due to silicon spheres for confocal and wide-field microscopy.

\section{Power dependence of $\gamma_{\mathrm{zpl}}, \lambda_{\mathrm{zpl}}$ and $A_{\mathrm{zpl}}$}

The change of the ZPL peak position and full width at half maximum (FWHM) and relative amplitude as a function of excitation laser power inducing direct heating of the nanodiamonds is shown in Fig. S3. The gradients of the peak shifting and broadening of ZPL are $9.3 \pm 0.3$ $\mathrm{nm} / \mathrm{W}$ and $48 \pm 1 \mathrm{~nm} / \mathrm{W}$, respectively. The broadening of the ZPL is about 5 times larger than the shift of the ZPL. Fig. S3b shows a linear dependence of amplitude of ZPL/PSB (PSB: phonon side band) against excitation power which has a gradient of $26.7 \pm 0.7 \mathrm{~W}^{-1}$. We investigated 9 nanodiamonds and the temperature sensitivities of ZPL FWHM and relative amplitude against ZPL position are presented in Table S1. 

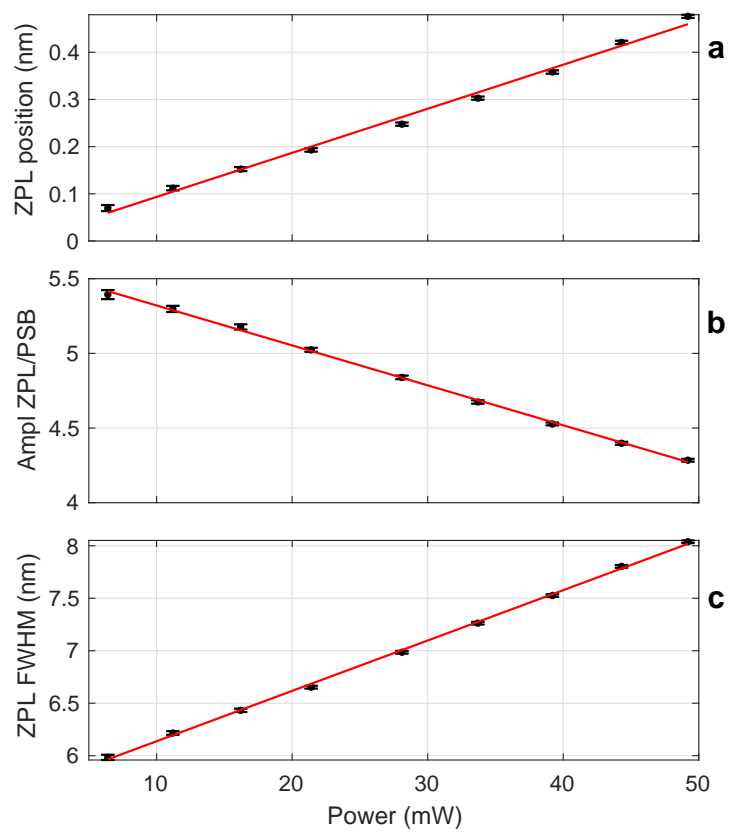

Figure S3: Dependence of ZPL position (a), amplitude of ZPL/PSB (b), and ZPL FWHM (c) on the excitation power. The solid lines show a least squares fits of straight lines to the experimental points.

\section{Laser spot-size measurement}

The spot size is derived from the fitting $\exp \left(-r^{2} / r_{0}^{2}\right)$ to the data, where $r$ is the distance from the center of the spot (and the location of the investigated crystals) and the best fit gives $r_{0}=1.2 \mu \mathrm{m}$.

\section{Laser heating confocal and wide-field}

To compare the heating effect due to silicon spheres, we recorded the change of ZPL position and FWHM with different power density for confocal and wide-field microscopy (Fig. S5). As can be seen from the figure, the slope of the ZPL position and FWHM using confocal is significantly less than that using wide-field microscopy. This means that wide-field induces more heating from silicon spheres due to the large spot size. By reducing the spot size of the excitation source, heating from the silicon spheres becomes less dominant. 
Table S1: Temperature sensitivities of ZPL linewidth and ZPL/PSB amplitude relative to the temperature sensitivity of the ZPL position (std: standard deviation)

\begin{tabular}{|l|l|l|}
\hline Sample no. & $\frac{\Delta \gamma_{\mathrm{zpl}}}{\Delta \lambda_{\mathrm{zpl}}}$ & $\frac{\Delta A_{\mathrm{zpl}}}{\Delta \lambda_{\mathrm{zpl}}}\left[\frac{1}{\mathrm{~nm}}\right]$ \\
\hline 1 & $4.4 \pm 0.1$ & $-2.5 \pm 0.2$ \\
\hline 2 & $4.88 \pm 0.07$ & $-2.66 \pm 0.09$ \\
\hline 3 & $6.6 \pm 0.6$ & $-3.5 \pm 0.3$ \\
\hline 4 & $6.5 \pm 0.8$ & $-3.1 \pm 0.5$ \\
\hline 5 & $3.7 \pm 0.2$ & $-2.6 \pm 0.2$ \\
\hline 6 & $6.2 \pm 0.6$ & $-2.6 \pm 0.5$ \\
\hline 7 & $4.5 \pm 0.5$ & $-2.5 \pm 0.3$ \\
\hline 8 & $3.8 \pm 0.2$ & $-2.05 \pm 0.09$ \\
\hline 9 & $5.1 \pm 0.2$ & $-2.8 \pm 0.2$ \\
\hline \multirow{2}{*}{ Distribution } & Mean: 5.1 & Mean: -2.7 \\
\cline { 2 - 3 } & std: 1.1 & std: 0.4 \\
\hline
\end{tabular}



Figure S4: Laser spot size measurement. Blue circles present luminescence intensity of a nanocrystal versus distance of the crystal from the center of the laser spot and the red solid line is fitting function. 
a

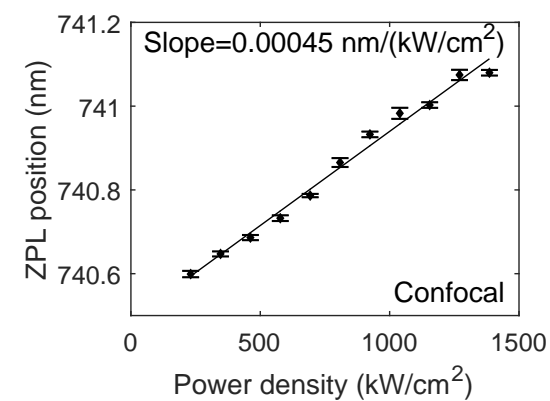

C

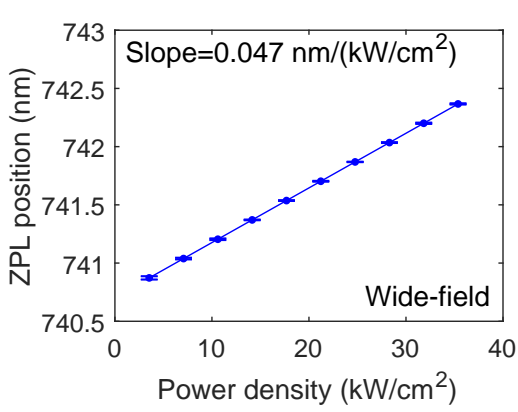

b
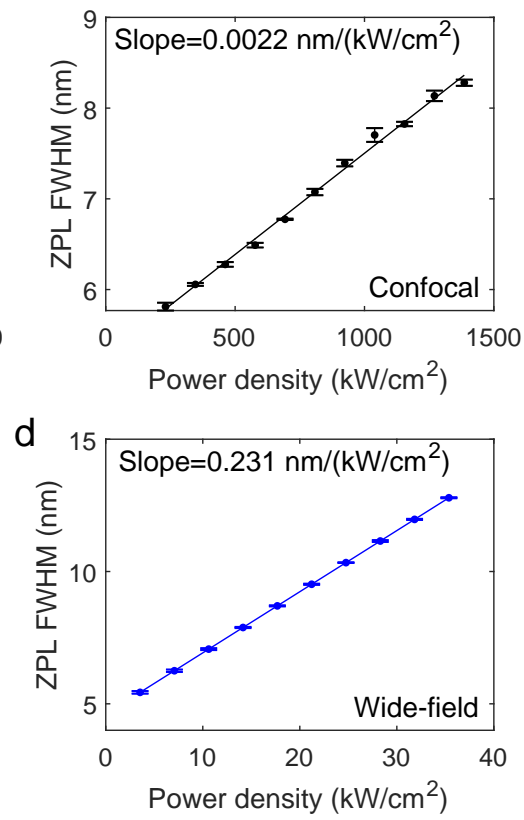

Figure S5: Comparison of heating effect between confocal and wide-field microscopy. Change of the ZPL position and FWHM against power density for $(\mathbf{a}, \mathbf{b})$ confocal and $(\mathbf{c}, \mathbf{d})$ wide-field microscopy. The gradients of ZPL position and FWHM against power density are about 100 times larger with wide-field microscopy.

\section{Example of correlation in two-parametric sensing}

Fig. S6 shows the presence of significant positive correlation between ZPL width and the splitting between the two Lorentzians $\left(\Delta_{\lambda}=\lambda_{\mathrm{zpl}}-\lambda_{\mathrm{psb}}\right)$. This correlation increases marginal fluctuation of the width which effectively "borrows" fluctuations from much larger fluctuations of the splitting. To remove this correlation which is an artifact of the fitting procedure between two fitting parameters, two new variables can be introduced. The variables can be defined as

$$
\begin{aligned}
& G_{x} \equiv \gamma \cos \phi-\Delta_{\lambda} \sin \phi \\
& G_{y} \equiv \gamma \sin \phi+\Delta_{\lambda} \cos \phi
\end{aligned}
$$

where $\gamma$ is the full-width of half maximum (FWHM) of ZPL, $\Delta_{\lambda}$ is the splitting between the ZPL and PSB, and $\phi$ is the angle of rotation required to tilt the elongated ellipse into a 
vertical orientation. The scatter plot for $G_{x}$ and $G_{y}$ is also shown Fig. S6. In this case the value of the angle is about 0.24 .

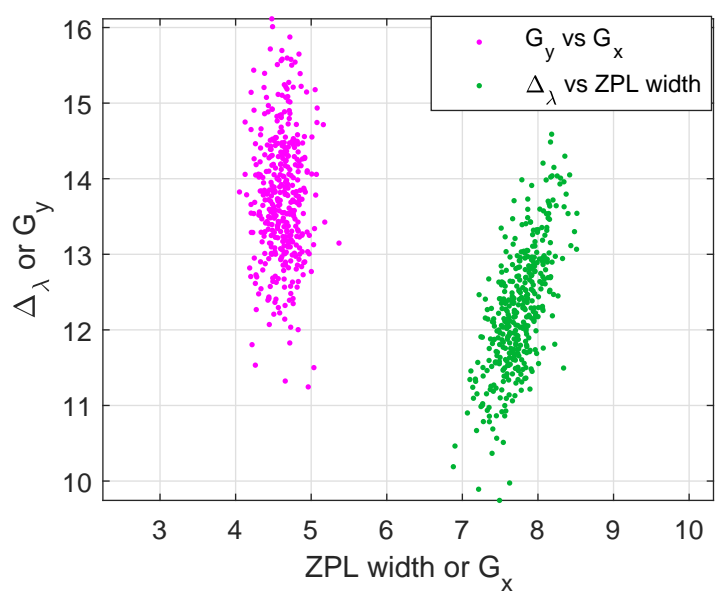

Figure S6: Correlation between fitting parameters. The simulated data points representing the splitting between the ZPL and PSB position against ZPL linewidth are shown in green dots. It demonstrates that there is a correlation caused by the fitting procedure. This correlation can be eliminated if two new variables are introduced and the scatter plot for these new variables is shown in pink dots. The standard deviation of $G_{x}$ distribution is a factor of 0.7 smaller than the standard deviation of $\gamma$.

\section{Examples of $G_{3}$-sensing with $\mathrm{SiV}$-centers}

Four different nanodiamonds were investigated using multiparametric data analysis and results are presented in Fig. S7. The values of noise floors are obtained from $14.2 \mathrm{mK} \mathrm{Hz}^{-1 / 2}$ to $18.6 \mathrm{mK} \mathrm{Hz}^{-1 / 2}$. Compared to the single-parameter sensing, the noise floor improves a factor of 2.7 to 3.8 .

We investigated that ZPL position, relative amplitude, and FWHM of the SiV spectrum are changed linearly with temperature. As long as this linearity keeps constant, the temperature sensitivity has not been changed. However, when the linear relationship between temperature and these parameters breaks down which happens above $340 \mathrm{~K},{ }^{1}$ the value of the temperature sensitivity also increases. The thermal sensitivity against temperature is shown in Fig. S8. The value of the thermal sensitivity is constant from $315 \mathrm{~K}$ to $340 \mathrm{~K}$. 
a

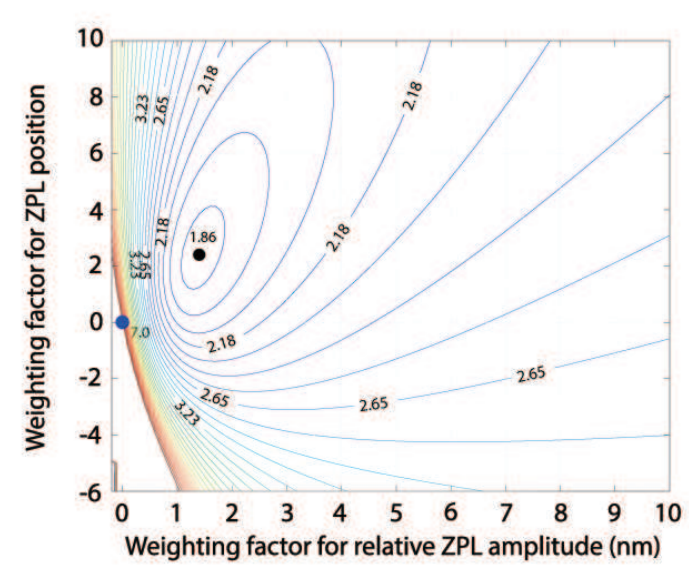

C

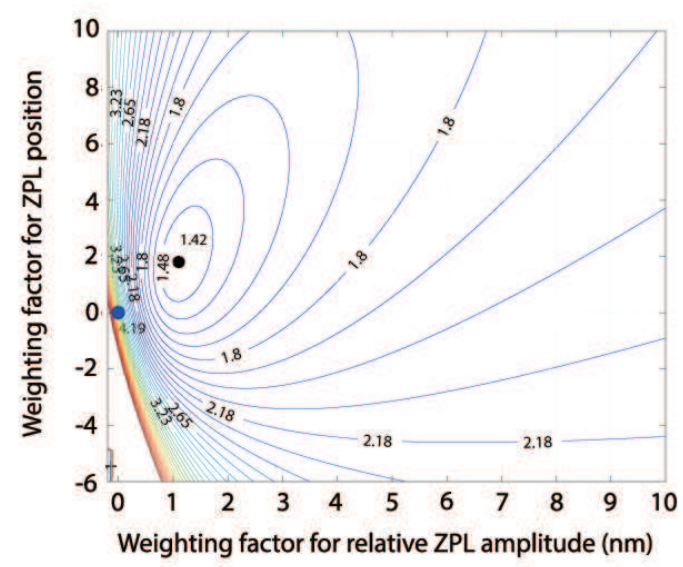

b

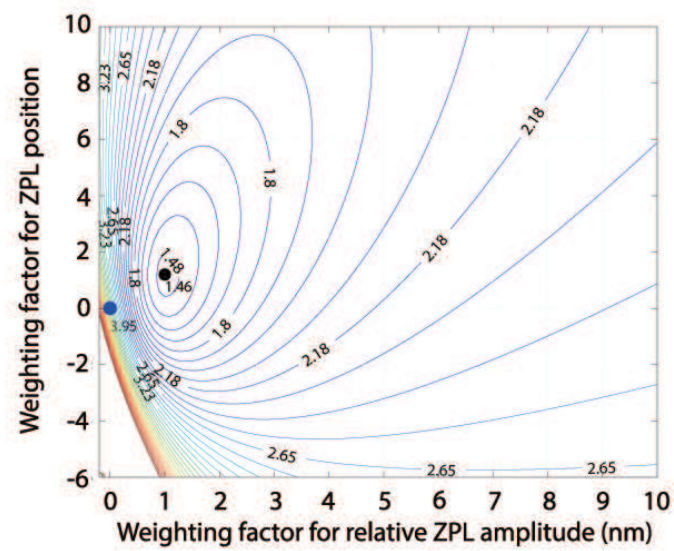

d

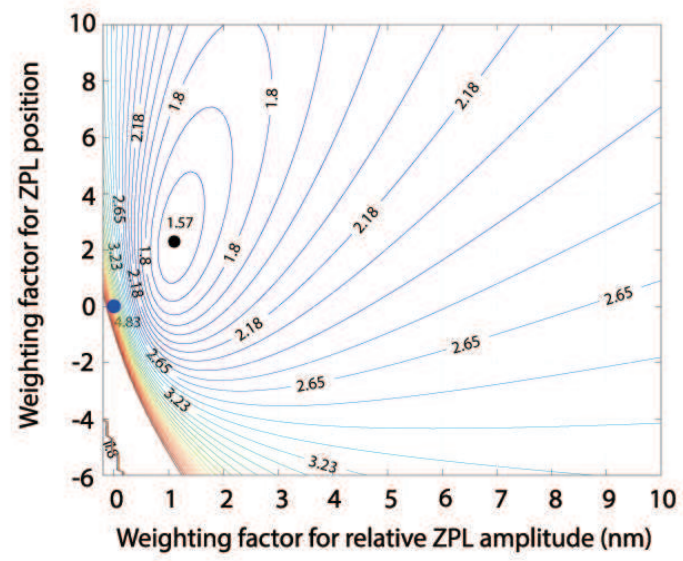

Figure S7: Multiparametric sensing with 4 different nanodiamonds. The blue dot corresponds to the noise floor using single parametric sensing $\left(w_{\lambda}=0\right.$ and $\left.w_{\mathrm{A}}=0\right)$, and the black dot is the noise floor using multiparametric analysis. The values of the noise floor are (a) 18.6 $\mathrm{mK} \mathrm{Hz}^{-1 / 2}$, (b) $14.6 \mathrm{mK} \mathrm{Hz}^{-1 / 2}$, (c) $14.2 \mathrm{mK} \mathrm{Hz}^{-1 / 2}$ and (d) $15.7 \mathrm{mK} \mathrm{Hz}^{-1 / 2}$ 


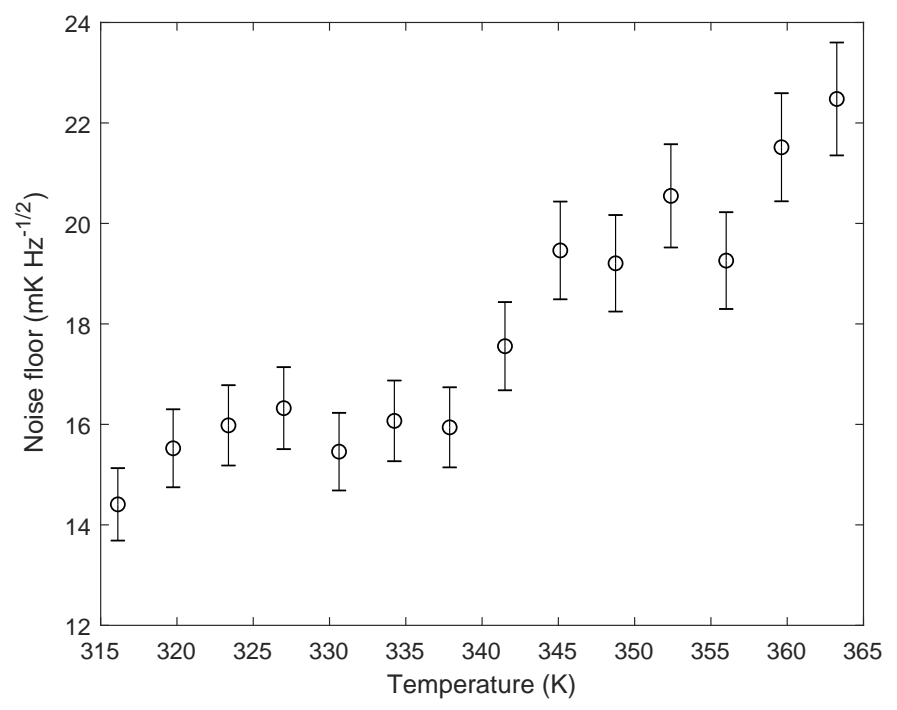

Figure S8: Change of the noise floor with temperature.

\section{References}

(1) Lagomarsino, S.; Gorelli, F.; Santoro, M.; Fabbri, N.; Hajeb, A.; Sciortino, S.; Palla, L.; Czelusniak, C.; Massi, M.; Taccetti, F., et al. Robust luminescence of the silicon-vacancy center in diamond at high temperatures. AIP Advances 2015, 5, 127117 